\title{
Localization of Point Sources for Systems Governed by the Wave Equation
}

\author{
Zafer Dogan ${ }^{a, b}$, Vagia Tsiminaki ${ }^{a, b}$, Ivana Jovanovic ${ }^{a, b}$, \\ Thierry Blu ${ }^{c}$, Dimitri Van De Ville ${ }^{a, b}$ \\ ${ }^{a}$ École Polytechnique Fédérale de Lausanne, Switzerland; \\ ${ }^{b}$ University of Geneva, Switzerland; \\ ${ }^{c}$ The Chinese University of Hong Kong, China
}

\begin{abstract}
Analytic sensing has recently been proposed for source localization from boundary measurements using a generalization of the finite-rate-of-innovation framework. The method is tailored to the quasi-static electromagnetic approximation, which is commonly used in electroencephalography. In this work, we extend analytic sensing for physical systems that are governed by the wave equation; i.e., the sources emit signals that travel as waves through the volume and that are measured at the boundary over time. This source localization problem is highly ill-posed (i.e., the unicity of the source distribution is not guaranteed) and additional assumptions about the sources are needed. We assume that the sources can be described with finite number of parameters, particularly, we consider point sources that are characterized by their position and strength. This assumption makes the solution unique and turns the problem into parametric estimation. Following the framework of analytic sensing, we propose a two-step method. In the first step, we extend the reciprocity gap functional concept to wave-equation based test functions; i.e., well-chosen test functions can relate the boundary measurements to generalized measure that contain volumetric information about the sources within the domain. In the second step - again due to the choice of the test functions - we can apply the finite-rate-of-innovation principle; i.e., the generalized samples can be annihilated by a known filter, thus turning the non-linear source localization problem into an equivalent root-finding one. We demonstrate the feasibility of our technique for a 3-D spherical geometry. The performance of the reconstruction algorithm is evaluated in the presence of noise and compared with the theoretical limit given by Cramer-Rao lower bounds.
\end{abstract}

Keywords: Analytic Sensing, Acoustic Imaging, Inverse Problem, Source Imaging, Source Localization, Wave Equation, Parameter Estimation

\section{INTRODUCTION}

Source localization from boundary measurements is an important type of inverse problem encountered in different fields. Such inverse problems require a mathematical model of the system and a physical background linking the parameters of the model to the measurements. Recently, analytic sensing has been proposed to solve the source localization problem from boundary measurements in systems governed by Poisson equation; ${ }^{1}$ e.g., as encountered in electroencephalography (EEG). In this work, we extend the analytic sensing method for wave-governed systems such as acoustic imaging. In photo/thermo-acoustic imaging, absorbed energy leads to thermoelastic expansion of the tissue and thus to generation of an equivalent acoustic source distribution. ${ }^{2}$ The motivation behind this work is to propose a non-iterative reconstruction method for the localization of some source distribution that could be characterized with finite number of position and intensity parameters from the measurements taken at the boundary of the observed region.

The major difficulty in such localization problem from boundary measurements arise from the fact that the problem is ill-posed; i.e., different values of the model parameters may yield the same measurement on the boundary. In order to overcome this ambiguity, based on the a priori assumption on the model, the source

Further author information: (Send correspondence to Z.D.)

Z.D.: E-mail: zafer.dogan@epfl.ch, Telephone: +41 216930610

Wavelets and Sparsity XIV, edited by Manos Papadakis, Dimitri Van De Ville, Vivek K. Goyal, Proc. of SPIE Vol. 8138, 81380P - @ 2011 SPIE · CCC code: 0277-786X/11/\$18 doi: $10.1117 / 12.894645$

Proc. of SPIE Vol. 8138 81380P-1 
distribution is parametrized in a way to acquire a well-posed problem. Moreover, it is also common to put some hypothesis about the properties of the source distribution. With these additional assumptions, the unicity of the solution is guaranteed.

In general, there are two ways of parametrizing the source distribution in these problems, namely the underdetermined and the overdetermined source models. In the former, the exact number of generating sources is unknown and, therefore, the solution space is discretized for fixed point solutions and the problem reduces to finding the corresponding intensities for the solution points. Therefore this model necessitates more priori assumptions to find the optimal solution, if possible, or most likely solution. Addition of prior information leads to different types of regularization methods. ${ }^{3-5}$ In the latter, the number of generating source points is assumed to be small and the localization problem turns into a parameter fitting problem that is nonlinear in the positions. ${ }^{6,7}$ Generally, there are two types of approaches for the second group of source modeling, namely multiple signal classification, i.e., MUSIC-type algorithms, ${ }^{8,9}$ and the application of the reciprocity gap concept. ${ }^{10}$

In this work, our goal is to device a parametric estimation of the underlying overdetermined (sparse) source model in such a way to extend and connect the source localization problem based on the Poisson and wave equations. The previous work ${ }^{1}$ on EEG source localization was based on the the Poisson equation that can be seen as a special case of wave equation for a wavenumber of $k=0$. We propose a parameter-fitting method connecting the two problem settings. The method consists of two principles. First, the sensing principle extracts the volumetric information from boundary measurements with a careful selection of some test functions. Second, the annihilation principle leads to a non-iterative solution to non-linear localization problem inspired from finiterate-of-innovation. With these principles the details of which will be provided in the sequel, we obtain a noniterative solution for the nonlinear source localization problem.

The outline of the paper is as follows. In Section 2, we define the problem statement and the parametrization of the source distribution that is necessary to render the problem well-posed. In Sections 3 and 4 , the two principles forming the proposed reconstruction algorithm are considered. And, in Section 5, we address the model mismatch and robustness to noise of the proposed model. The experimental results for a single and multi source localization problems will be provided in Section 6 . And, we conclude the with a short discussion on our future research.

\section{PROBLEM SETTING}

In this paper, we focus on the recovery of pointwise acoustic sources from the boundary acoustic field pressure measurements. Let us consider a volume $\Omega$ with a boundary $\partial \Omega$. Moreover, we assume an acoustic source distribution $p$ within the volume $\Omega$. The acoustic sources inside the volume $\Omega$ will create an acoustic pressure on the boundary $\partial \Omega$ according to the wave equation ${ }^{11,12}$ such that

$$
\Delta u(\mathbf{r}, t)-\frac{1}{c^{2}} \frac{\partial^{2} u(\mathbf{r}, t)}{\partial t^{2}}=p(\mathbf{r}, t),
$$

where $u(\mathbf{r}, t)$ is the sound pressure measured at location $\mathbf{r} \in \mathbb{R}^{3}$ and time $\mathrm{t}, p(\mathbf{r}, t)$ is the acoustic source term, $c$ is the sound speed which is assumed to be constant and $\Delta$ is the spatial Laplacian operator. In the temporal frequency domain, the wave equation will yield the inhomogeneous Helmholtz equation

$$
\Delta U+k^{2} U=P
$$

where $U$ and $P$ are the acoustic pressure field and the source term in temporal frequency domain, respectively, and $k$ is the wave number such that $k^{2}=\frac{\omega^{2}}{c^{2}}$, where $\omega$ is the angular frequency of the wave.

In order to have a unique solution for the forward problem (i.e., given a source term $P$ with a compact support to solve the inhomogeneous Helmholtz equation uniquely) one needs to specify a boundary condition at infinity, typically Sommerfeld radiation condition. Under this assumption, the solution for the acoustic pressure field is given as the convolution of the source term with the corresponding Green's function of the problem. The Green's function for Helmholtz equation which satisfies:

$$
\left(\Delta+k^{2}\right) G\left(\mathbf{r}, \mathbf{r}^{\prime}\right)=\delta\left(\mathbf{r}-\mathbf{r}^{\prime}\right)
$$


in unbounded space is an outgoing spherical wave given as follows:

$$
G(\mathbf{r})=\frac{e^{i k|\mathbf{r}|}}{4 \pi|\mathbf{r}|}
$$

In this case, the solution to the inhomogeneous Helmholtz Eq. (2) in $\mathbb{R}^{3}$ with the specified boundary condition at infinity is given as

$$
U(\mathbf{r})=(G * P)(\mathbf{r})=\int_{\mathbb{R}^{3}} G\left(r-r^{\prime}\right) P\left(r^{\prime}\right) d r^{\prime} .
$$

At this point, one could determine the acoustic pressure field assuming a source distribution enclosed by the volume, $\Omega$. However, the problem that is going to be attacked, here, is to determine a source distribution that creates acoustic pressure field on the surface of the volume, $\partial \Omega$. Thus, the problem is as stated as follows:

$\mathcal{P}$ : Knowing the pressure field only on the boundary by $\left.U\right|_{\partial \Omega}$ and $\left.\nabla U\right|_{\partial \Omega}$, find the enclosed acoustic source distribution within volume $\Omega$ such that Eq. (2) is satisfied.

The above stated problem $\mathcal{P}$ is not well-posed since there are many solutions satisfying the measurement set of the acoustic pressure field on the surface of the volume. Therefore, in order to have a well-posed question and a corresponding unique solution it is necessary to have some additional assumptions.

For this purpose, we need to further parametrize the source distribution; i.e., the source term is assumed to consist of pointwise acoustic sources, such that

$$
P(\mathbf{r})=\sum_{m=1}^{M} c_{m} \delta\left(\mathbf{r}-\mathbf{r}_{m}\right),
$$

where $c_{m} \in \mathbb{R}, M \in \mathbb{N}_{0}$ and $\mathbf{r}_{m} \in \Omega$. With this parametrization, the source term is completely characterized by the positions and intensities of $M$ sources (with $4 \mathrm{M}$ parameters). Hence, we will see that with this setting the problem can be casted into a typical setting for finite rate of innovation ${ }^{13,14}$ and the problem $\mathcal{P}$ becomes wellposed $^{15}$ where the solution algorithm to $\mathcal{P}$ will be provided in the following sections. Consequently, the nonlinear problem of recovering sparse sources from boundary measurements will turn into an equivalent parameter fitting problem to acquire the positions and the intensities of the pointwise acoustic sources.

\section{SENSING PRINCIPLE}

In this section, the sensing principle is developed based on divergence theorem to extract volumetric information from the boundary measurements. For this purpose, we provide a family of test functions to retrieve the generalized samples of the unknown source distribution.

THEOREM 1. Let $U$ be the acoustic pressure field generated by some source distribution $P$ in $\Omega$, satisfying Eq. (2), if we choose a test function $\Psi$ such that $\Delta \Psi=-k^{2} \Psi$ within $\Omega$, the scalar product $\langle\Psi, P\rangle$ can be stated as a surface integral :

$$
\langle\Psi, P\rangle=\oint_{\partial \Omega}(\Psi \nabla U-U \nabla \Psi) \cdot \mathbf{e}_{\partial \Omega} d S
$$

where $\nabla$ is the gradient operator and $\mathbf{e}_{\partial \Omega}$ is the outward normal vector on the surface of $\Omega$. Here, the scalar products $\langle\Psi, P\rangle$ can be seen as generalized samples of the unknown distribution $P$.

Proof. For any twice differentiable functions on a volume $\Omega, U$ and $\Psi$, the second Green's identity states that the following relationship between the volume and surface integrals hold:

$$
\int_{\Omega}\left(U \nabla^{2} \Psi-\Psi \nabla^{2} U\right) d V=\oint_{\partial \Omega}(U \nabla \Psi-\Psi \nabla U) \cdot \mathbf{e}_{\partial \Omega} d S
$$

This relationship allows to relate the boundary measurements of the pressure field to the generalized samples of the unknown distribution that contain volumetric information about the sources. In particular, with the above choice of the test function, we obtain the proposed generalized samples as follows: 


$$
\begin{aligned}
\langle\Psi, P\rangle & =-\int_{\Omega}\left(U\left(-k^{2} \Psi\right)-\Psi\left(P-k^{2} U\right)\right) d V \\
& =-\oint_{\partial \Omega}(U \nabla \Psi-\Psi \nabla U) \cdot \mathbf{e}_{\partial \Omega} d S
\end{aligned}
$$

Note that, any test function $\Psi$ satisfying the relation $\Delta \Psi=-k^{2} \Psi$ within $\Omega$ can be chosen in order to obtain the generalized samples of the source term.

\section{ANNIHILATION PRINCIPLE}

The main idea in this section is to select a proper test function and to develop a non-iterative reconstruction algorithm for the nonlinear source localization problem satisfying the inhomogeneous Helmholtz equation.

A complex-valued function $\psi$ that is defined on open subset $\mathrm{G}$ of $\mathbb{C}$ and differentiable at every point of $\mathrm{G}$ is said to be analytic in its domain. Moreover, any analytic function of class $\mathbb{C}^{2}$ is harmonic; i.e., satisfies Laplace's equation by Cauchy-Rieman equations. ${ }^{16}$ Considering the localization problem for systems governed by Poisson's equation as encountered in EEG, ${ }^{1,17}$ the proposed test functions are analytic rational functions of special type. We extend the same idea for systems governed by wave equation as follows:

For any analytic function $\psi$, we state the following family of functions

$$
\Psi(x, y, z)=e^{ \pm i k z} \psi(x, y)
$$

satisfies the relation, $\nabla^{2} \Psi+k^{2} \Psi=0$. Hence, for the source parametrization as in Eq. (6), the following family of test functions is proposed:

$$
\Psi_{n}(x, y, z)=\frac{e^{i k z}}{x+i y-a_{n}}, \quad a_{n} \notin \Omega,
$$

where $\psi(x, y)=\frac{1}{x+i y-a_{n}}$ is the analytic part of the proposed test function, $a_{n}=a e^{i n \alpha}$ with the radius $|a|$ is chosen such that $a_{n} \notin \Omega$ and $\left.\alpha \in\right] 0,2 \pi[$. In other words, the poles of the test functions lie excluding the domain of the enclosing the volume $\Omega$ at equidistant angles. Note that the angle $\alpha$ is completely arbitrary and $N \alpha$ do not need to be equal to $2 \pi$; i.e., the poles of the test functions could be located in a neighborhood at equidistant radial angles.

Based on the selected test function, localization of the acoustic sources will be done for the plane defined by the poles of the test function; i.e., XY-plane. First, let's define a polynomial, $Q(X)$ whose roots are the position of the acoustic sources on the XY-plane:

$$
Q(X)=\prod_{m=1}^{M}\left(X-s_{m}\right)=\sum_{k=0}^{M} q_{k} X^{k} \text { where } s_{m}=x_{m}+i y_{m} \text { with } q_{M}=1 .
$$

With this selection of the proposed family of test functions, the generalized samples of the source term parametrized as in Eq. 6 turns into an annihilable function as follows:

$$
\begin{aligned}
\left\langle\Psi_{n}, P\right\rangle=\mu_{n} & =\sum_{m=1}^{M} \frac{c_{m} e^{i k z_{m}}}{x_{m}+i y_{m}-a_{n}} \\
& =\frac{\sum_{m=0}^{M-1} c_{m}^{\prime} e^{i m n \alpha}}{\prod_{m=1}^{M}\left(x_{m}+i y_{m}-a_{n}\right)} \\
& =\frac{\sum_{m=0}^{M-1} c_{m}^{\prime} e^{i m n \alpha}}{Q\left(a_{n}\right)},
\end{aligned}
$$


where $c_{m}^{\prime}$ are complex-valued coefficients that do not depend on $n$ nor $\alpha$. The sequence $u=\left\{u_{n}\right\}$ for $n \in$ $\llbracket 0, N-1 \rrbracket$, whose coefficients are defined by $u_{n}=Q\left(a_{n}\right) \mu_{n}$, can be annihilated by an FIR digital filter $h=$ $\left\{h_{k}\right\}$ for $k \in \llbracket 0, N-1 \rrbracket$ characterized as follows:

$$
H(z)=\sum_{k \in \mathcal{Z}} h_{k} z^{-k}=\prod_{k=0}^{M-1}\left(1-e^{i k \alpha} z^{-1}\right)
$$

The filter $h$ that has its zeros at $e^{i k \alpha}$ for $k \in \llbracket 0, M-1 \rrbracket$ annihilates the sequences $u$ as follows:

$$
h * u=0, \forall n \in \llbracket M, N-1 \rrbracket .
$$

THEOREM 2. The coefficients of the polynomial $Q(X)$ defined by Eq. (12) satisfy the following linear system of equations:

$$
\sum_{k=0}^{M} A_{n, k} q_{k}=0, \quad A_{n, k}=\sum_{n^{\prime}=0}^{N-1} h_{n-n^{\prime}} a_{n^{\prime}}^{k} \mu_{n^{\prime}}
$$

where $n=M, \ldots, N-1$. Solving the linear system expressed as $\mathbf{A} Q=0$, the source positions would be roots of the polynomial $Q$.

Proof. From the annihilation equation,

$$
\begin{aligned}
0=\{h * u\}_{n} & =\sum_{n^{\prime}=0}^{N-1} h_{n-n^{\prime}} Q\left(a_{n^{\prime}}\right) \mu_{n^{\prime}} \\
& =\sum_{n^{\prime}=0}^{N-1} h_{n-n^{\prime}} \sum_{k=0}^{M} q_{k} a_{n^{\prime}}^{k} \mu_{n^{\prime}} \\
& =\sum_{k=0}^{M} q_{k} \sum_{n^{\prime}=0}^{N-1} h_{n-n^{\prime}} a_{n^{\prime}}^{k} \mu_{n^{\prime}} \\
& =\sum_{k=0}^{M} A_{n, k} q_{k} .
\end{aligned}
$$

Combining the two theorems, we end up with a one step solution for localization of the acoustic sources with the acoustic pressure field measured only on the boundary. Solving Eq. (17), the coefficients of the polynomial $Q$ are obtained and thus the roots of it which are the locations of the sources on the XY-plane, by definition. The third location components and the intensities of the source points can be computed as follows with the available generalized samples and the estimated locations of the sources:

$$
\underbrace{\left\langle\Psi_{n}, P\right\rangle=\mu_{n}}_{\text {known }}=\sum_{m=1}^{M} \frac{c_{m} e^{i k z_{m}}}{\underbrace{x_{m}+i y_{m}}_{\text {estimated locs. }}-a_{n}} .
$$

In matrix notation, we can express this system as $\mathcal{B} \mathbf{p}=\mathbf{r}$, where $\mathcal{B}$ is an $N \times M$ matrix with the entries $\mathcal{B}_{m, n}=\frac{1}{x_{m}+i y_{m}-a_{n}}$, and $\mathbf{p}$ is an $M \times 1$ vector with entries $p_{m}=c_{m} e^{i k z_{m}}$ and $\mathbf{r}$ is an $N \times 1$ vector with entries $r_{n}=\mu_{n}$. The performance of the solution of the above linear system for the third component of the locations of the source term depends on the selection of the wavenumber due to the periodicity of the exponential term. Therefore, choosing the proper period for the $z_{m}$ is achieved with the minimization of the error in the reconstructed pressure field on the surface as follows:

$$
\hat{z}_{m}=\arg \min _{z_{m}(l)}\left\|V_{\partial \Omega}-V_{\partial \Omega}\left(\hat{x}_{m}, \hat{y}_{m}, z_{m}(l)\right)\right\|, \text { where } z_{m}(l)=\frac{\phi+2 \pi l}{k}, l \in \mathbb{Z},
$$

where $\hat{x}_{m}, \hat{y}_{m}$ are the previously estimated locations. 


\section{MODEL MISMATCH}

In terms of matrix representation, Eq. (16) can be formulated as follows:

$$
\underbrace{\mathbf{H} \mu \mathbf{a}}_{\mathbf{A}} Q=0,
$$

where $\mathbf{H}$ is an $(N-M) \times N$ Toeplitz Matrix representing the digital filter $h, \mu$ is $N \times N$ diagonal matrix containing the generalized samples, $\mathbf{a}$ is $N \times(M+1)$ Vandermonde matrix of poles $a_{n}$ and $Q$ is a vector of $\mathrm{M}+1$ polynomial coefficients with the following explicit representations:

$$
\mathbf{H}=\left[\begin{array}{cccccc}
h_{M} & \ldots & h_{0} & 0 & \ldots & 0 \\
0 & h_{M} & \ldots & h_{0} & \ldots & 0 \\
. & \cdot & . & . & . & . \\
0 & \ldots & 0 & h_{M} & \ldots & h_{0}
\end{array}\right], \mu=\left[\begin{array}{cccc}
\mu_{0} & 0 & \ldots & 0 \\
0 & \mu_{1} & \ldots & 0 \\
. & \cdot & \cdot & \cdot \\
0 & . & \ldots & \mu_{N-1}
\end{array}\right], \mathbf{a}=\left[\begin{array}{ccc}
a_{0}^{0} & \ldots & a_{0}^{M} \\
\cdot & \cdot & \cdot \\
\cdot & \cdot & \cdot \\
a_{N-1}^{0} & \ldots & a_{N-1}^{M}
\end{array}\right]
$$

The system $\mathbf{A} Q=0$ has $(N-M)$ equations with $M$ unknowns polynomial coefficients since we assume to scale the coefficients such that $q_{M}=1$ as in Eq. (12). From here, we conclude that we need at least $N=2 M$ generalized samples to retrieve the $M$ polynomial coefficients. Moreover, for the noiseless case and for $M$ distinct source positions, the system matrix $\mathbf{A}$ is of rank $M$.

The algorithm explained so far assumes perfect data, but in practice the measured data is corrupted with measurement noise. In order to compensate for the measurement noise and any kind of model mismatch, we propose a modified Cadzow denoising algorithm ${ }^{18}$ where the summary of the denoising method is provided in Fig. 1 .

Let's assume that the generalized samples are corrupted with complex Additive White Gaussian Noise (AWGN); i.e., the in-phase and quadrature phase components of the noise are independent having zero mean and $\sigma^{2}$ variance. ${ }^{19}$ In the presence of this noise, the system matrix becomes

$$
\tilde{\mathbf{A}} Q=\mathbf{H} \tilde{\mu} \mathbf{a} Q=0,
$$

where $\tilde{\mu}$ represents the noisy generalized samples. In order to denoise the generalized samples, we propose the following two step denoising approach.

1) Preconditioning: replace $\tilde{\mathbf{A}}$ with $\tilde{\mathbf{A}}_{\mathbf{0}}=\mathbf{H}_{\mathbf{0}} \tilde{\mu} \mathbf{a}_{0}$

a) $\mathbf{H}$ with $\mathbf{H}_{\mathbf{0}}$ such that $\mathbf{H}=\mathbf{U S H}_{\mathbf{0}}$

b) $\mathbf{a}$ with $\mathbf{a}_{0}$ such that $\mathbf{a}=\mathbf{a}_{0} \mathbf{S V}^{*}$

2) Cadzow-like denoising iterations

a) Use higher number of sources $\tilde{M}$ to allow noise and model mismatch

b) Low-rank approximation $\hat{\mathbf{A}}$ (i.e., rank $M$ )

c) Get new generalized samples: $\hat{\mu}=\arg \min _{\mu}\left\|\mathbf{H}_{\mathbf{0}} \mu \mathbf{a}_{\mathbf{0}}-\hat{\mathbf{A}}\right\|_{F}$

Figure 1: Proposed denoising procedure with Cadzow flavor

The convolution matrix, $\mathbf{H}$ is in general not unitary, even if it has maximal rank. In case, the generalized samples are not known with high accuracy, this may distort the recovered polynomial coefficients. Hence, performing a singular value decomposition of $\mathbf{H}$, we first replace the convolution matrix with $\mathbf{H}_{\mathbf{0}}$ such that $\mathbf{H}=\mathbf{U S H}_{\mathbf{0}}$ to better condition the system matrix. Next, we observe that $\mathbf{A}_{\mathbf{0}}$ might be still ill-conditioned in case the angular distances between the singularities of the test pole locations are small. Therefore, we replace $\mathbf{a}$ by a unitary matrix $\mathbf{a}_{\mathbf{0}}$ obtained such that $\mathbf{a}=\mathbf{a}_{\mathbf{0}} \mathbf{S} \mathbf{V}^{*}$. The resulting system matrix, $\tilde{\mathbf{A}}_{\mathbf{0}}=\mathbf{H}_{\mathbf{0}} \tilde{\mu} \mathbf{a}_{\mathbf{0}}$ will be used to denoise the generalized samples. In Cadzow denoising iterations, we exploit the fact that the system matrix has to remain of rank $M$ at each iteration. Therefore, for any $L>M$, the last $L-M$ singular values of the $\tilde{\mathbf{A}}_{\mathbf{0}}$ 
are forced to zero to get a low rank approximation of the system matrix, $\hat{\mathbf{A}}$. The denoised signal is achieved as a minimization of the following objective:

$$
\tilde{\mu}=\arg \min _{\mu}\left\|\mathbf{H}_{\mathbf{0}} \mu \mathbf{a}_{\mathbf{0}}-\hat{\mathbf{A}}\right\|_{F},
$$

using a least squares fit with respect to the Frobenius norm, $\|.\|_{F}$. We iterate the scheme until the last $L-M$ singular values of $\hat{\mathbf{A}}$ are smaller than a given threshold (e.g., 10 ${ }^{-6}$ ). In Fig. 2, the proposed method is summarized. In the first step, we obtain generalized samples; i.e., the sensing step. In case these samples are noisy, we iterate through Cadzow-like denosing step. We annihilate the denoised generalized samples; i.e., the annihilation step, to find the XY-plane locations of the source source distribution. For the Z-locations and the intensities, we solve for another linear system to choose the proper period as explained in Eq. (18).

The performance of the proposed algorithm will be evaluated in the presence of noise and compared with the computed Cramer-Rao lower bound (CRLB) for the generalized samples with complex valued AWGN. ${ }^{20}$ For the computation of CRLBs, the noisy generalized samples have the following signal model:

$$
x[n]=s[n ; \boldsymbol{\Theta}]+v_{I}[n]+j v_{Q}[n], \quad n \in \llbracket 0, N-1 \rrbracket,
$$

where $v_{I}$ and $v_{Q}$ represents the in-phase and quadrature components of the noise; i.e., real and imaginary parts of the noise, respectively. We define

$$
s[n ; \boldsymbol{\Theta}]=\mu_{n}, \quad \boldsymbol{\Theta}=[\mathbf{x}, \mathbf{y}, \mathbf{z}, \mathbf{c}]^{T},
$$

with $\mathbf{x}=\left[x_{1}, \ldots x_{M}\right] \mathbf{y}=\left[y_{1}, \ldots y_{M}\right] \mathbf{z}=\left[z_{1}, \ldots z_{M}\right] \mathbf{c}=\left[c_{1}, \ldots c_{M}\right]$. Assuming the regularity condition is met, ${ }^{20}$ we define the Fisher Information Matrix (FIM), $I(\Theta)$ whose $i j^{t h}$ entry for $i, j=1,2, \ldots, 4 M$ is given by:

$$
[I(\boldsymbol{\Theta})]_{i j}=\frac{1}{\sigma^{2}} \sum_{n=0}^{N-1}\left[\operatorname{Re}\left\{\frac{\partial s[n ; \boldsymbol{\Theta}]}{\partial \Theta_{i}}\right\} \operatorname{Re}\left\{\frac{\partial s[n ; \boldsymbol{\Theta}]}{\partial \Theta_{j}}\right\}+\operatorname{Im}\left\{\frac{\partial s[n ; \boldsymbol{\Theta}]}{\partial \Theta_{i}}\right\} \operatorname{Im}\left\{\frac{\partial s[n ; \boldsymbol{\Theta}]}{\partial \Theta_{j}}\right\}\right] .
$$

The diagonal elements of inverse FIM yield the Cramer-Rao bounds for the elements of the parameter vector $\Theta$.

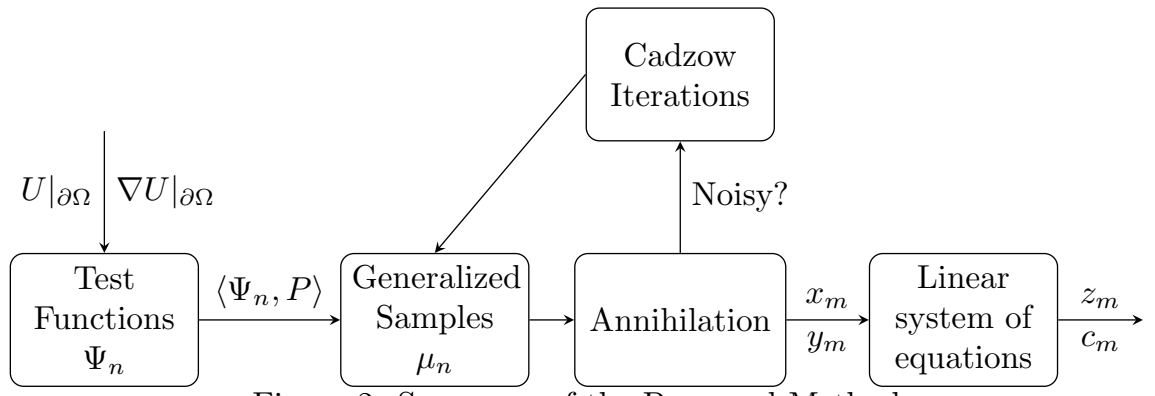

Figure 2: Summary of the Proposed Method

\section{EXPERIMENTAL RESULTS}

We provide the results of the proposed reconstruction algorithm using simulations of a monopole acoustic source. Under the signal model in Eq. (20), we assume the model mismatch is represented with a complex AWGN on the computed generalized samples. We focus on the localization rather than the intensity retrieval, therefore the intensities of the sources are assumed to be unity. Hence, we compare the retrieved localization errors for the projected plane; e.g., XY-plane here, with the theoretical limits which are obtained as the diagonal element of the inverse FIM given as in Eq. (21) for different noise levels of the generalized samples designated with the SNR of the signal as: 


$$
S N R=10 \times \log \frac{P_{s}}{P_{v}}=10 \times \log \frac{\sum_{n}|s[n ; \Theta]|^{2}}{\sum_{n}\left|v_{I}[n]+i v_{Q}[n]\right|^{2}} .
$$

The experimental setup is a sphere of radius $0.1 \mathrm{~m}$, the test functions are located on the XY-plane with equidistant angles enclosing the domain. The experiment is carried out at $100 \mathrm{KHz}$ which corresponds to a wavenumber of 418. The experimental setup in the XY-plane view is shown in Fig. 3 for a single acoustic source.

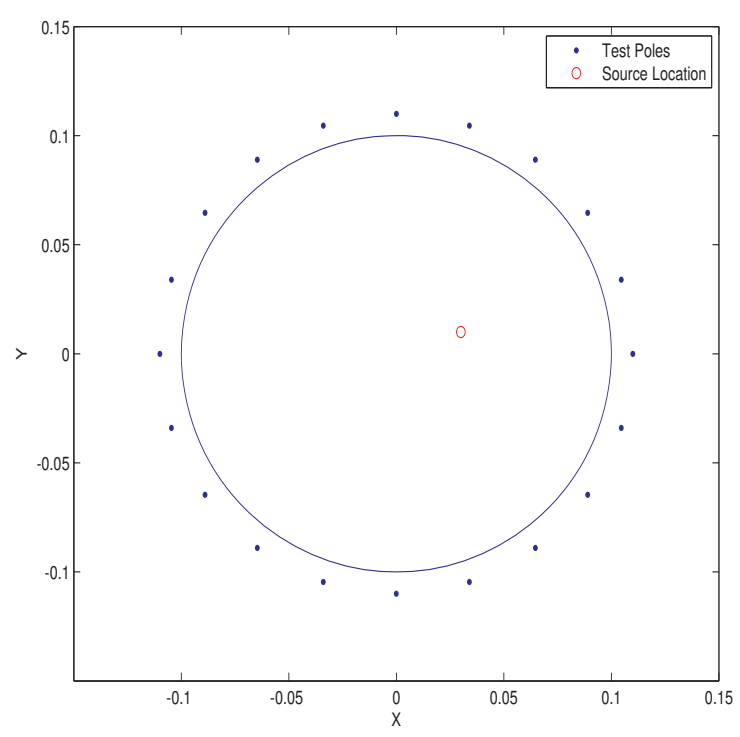

Figure 3: XY view of domain (sphere of radius 0.1) showing the poles of the test functions and one source location with unit intensity.

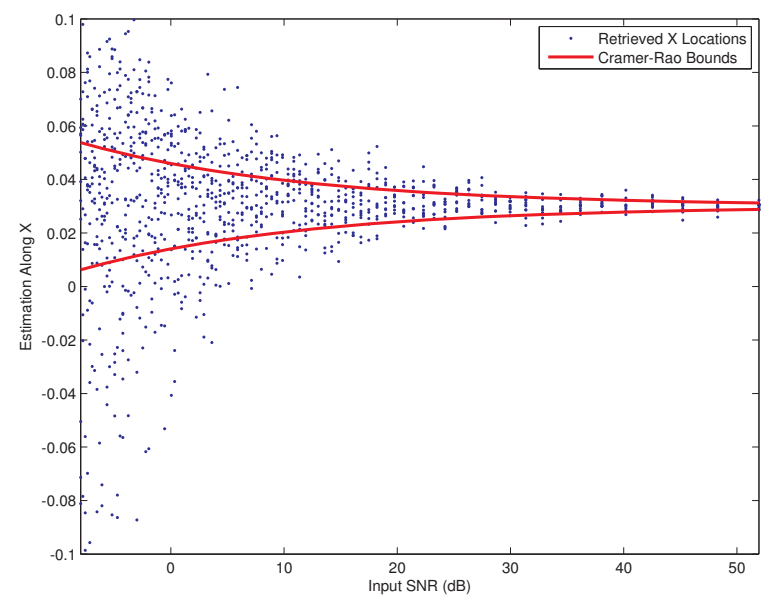

(a)

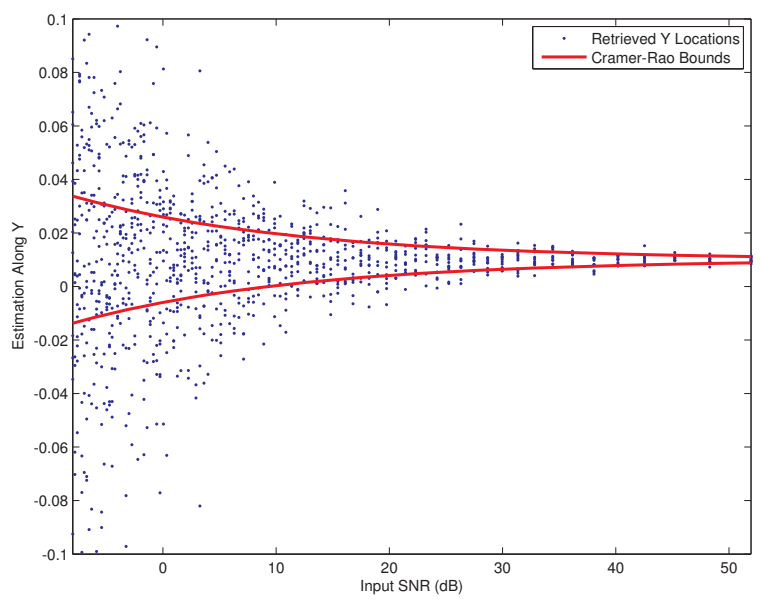

(b)

Figure 4: CRLBs (solid line) and the estimated location of the acoustic source (circles) with 20 test sensors: $\Psi_{n}=\frac{e^{i k z}}{x+i y-a_{n}}$, with $a_{n} \in \llbracket 0,19 \rrbracket$. (a) Scatter plot of estimation along X for 11 noisy samples (b) Scatter plot of estimation along $\mathrm{Y}$ for 11 noisy samples.

Moreover, in order to compensate for the model mismatch on the generalized samples, we make use of adapted Cadzow denoising iterations until we reach the threshold of $10^{-6}$ for the second singular value of the system matrix, where the details denoising scheme can be found in Sec. 4. 


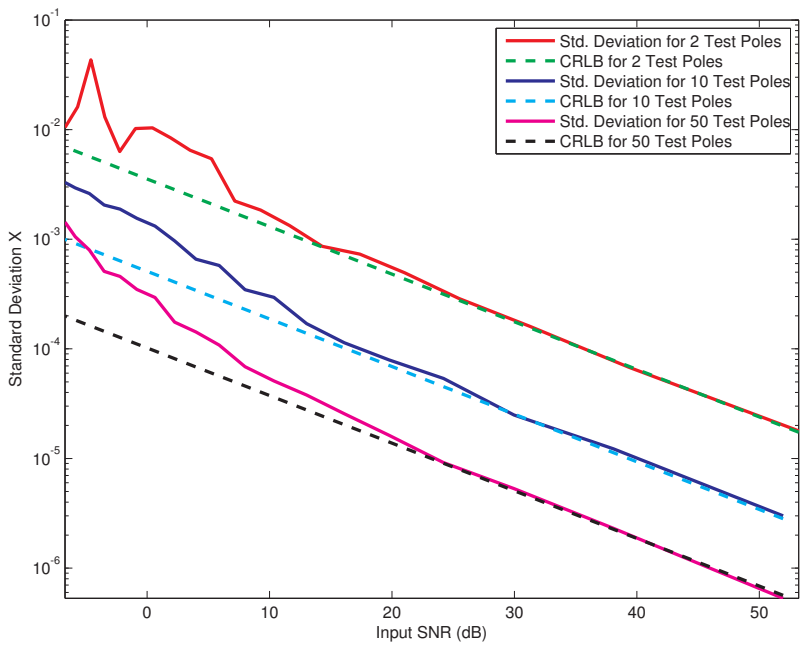

(a)

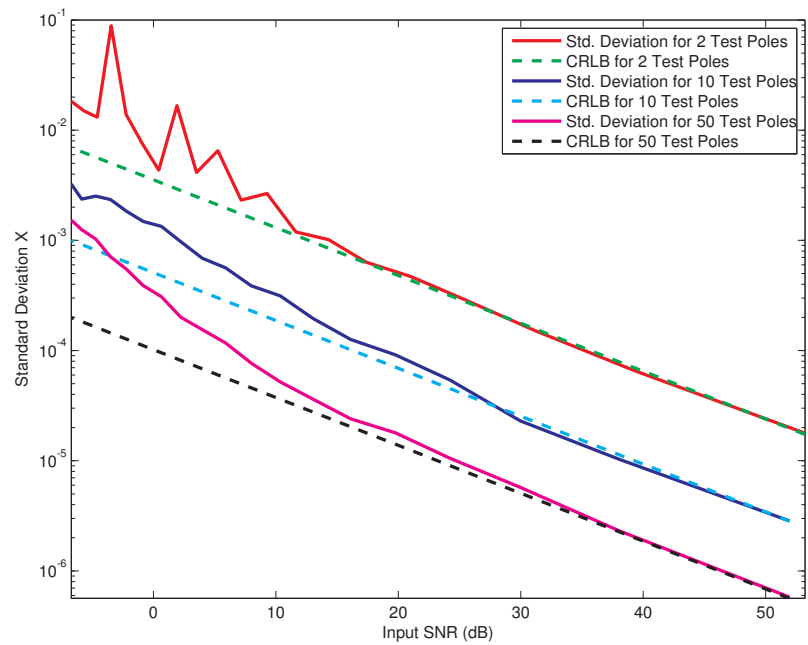

(b)

Figure 5: Observed standard deviation for 1000 runs compared against the CRLBs. (a) Estimation along X-axis (b) Estimation along Y-axis.

The selected test function for the current setup is given as:

$$
\Psi_{n}=\frac{e^{i k z}}{x+i y-0.11 e^{i n \alpha}}, \text { where } \alpha=2 \pi / N
$$

where $N$ is the number of generalized samples. The test poles are located at a radius of $0.11 \mathrm{~m}$; i.e., excluding the domain.

In Fig. 4, we show the results using the 20 generalized samples for the given setup. In Fig. 4 (a) and (b), the scatter plot of estimation along $\mathrm{X}$ and $\mathrm{Y}$ directions and the corresponding theoretical limits are provided. Here, we observe that the proposed method reaches the CRLBs for an SNR level of around 20dB in generalized samples.

In Fig. 5, we summarize the performance of the reconstruction algorithm comparing the observed standard deviation for 1000 realizations with the theoretical limit, plotted in log scale for different number of test functions. We clearly observe that the more number of generalized samples we have, the better localization we get. Moreover, the localization method achives the CRLB for SNR level of $20 \mathrm{~dB}$ in generalized samples of the signal. As the number of test functions increases from 2 to 50, it is important to notice that the method reaches the theoretical bound for generalized samples having SNR less than $20 \mathrm{~dB}$.

We also provide the localization performance of the proposed method for multiple source imaging. In Fig. 6, (a) and (b), the scatterplot of three source localization for XY-plane and XZ-plane are provided, respectively. Here, the z- axis solutions are chosen within a periodic set as the minimization of Eq. 18.

The preliminary results of the proposed algorithm demonstrate the feasibilty of the source localization for the nonlinear source imaging problem. Thanks to exploiting the system matrix properties in the adapted Cadzow denoising algorithm, the proposed method reaches the theoretical lower bound on the estimation parameters for a large range of SNR. The noniterative approach makes the solution computationally efficient and promising for future research.

\section{CONCLUSION}

We developed a non-iterative source localization algorithm for systems governed by the wave equation as an extension to the analytic sensors proposed recently for the Poisson's equation. We observe that for a particular 


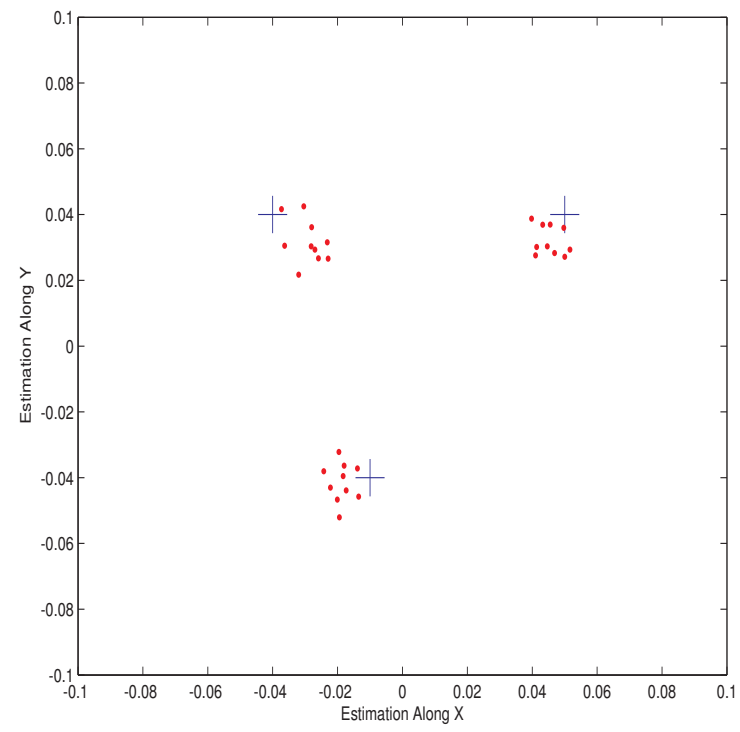

(a)

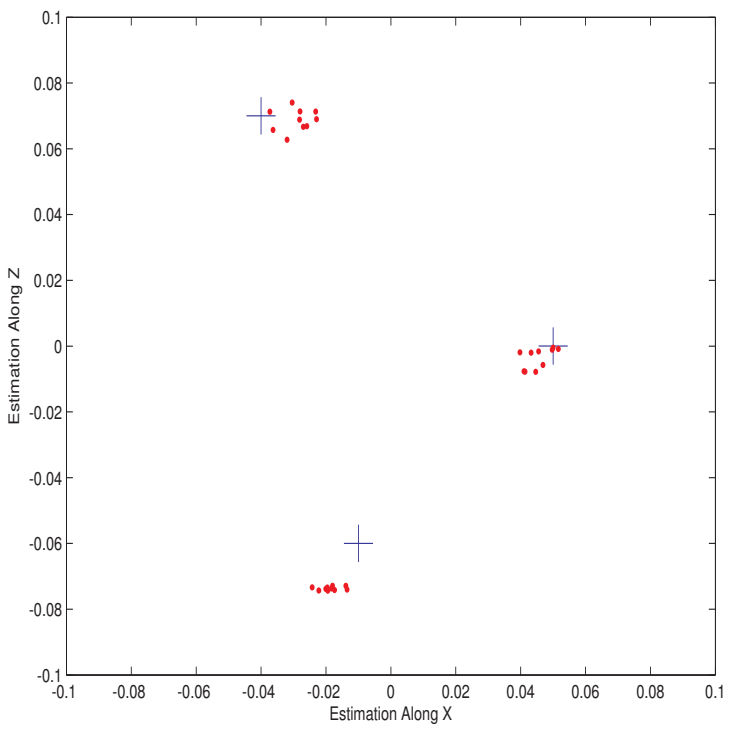

(b)

Figure 6: Scatterplot of multiple runs of source localization for three sources, $\mathrm{SNR}=20 \mathrm{~dB}$ and $N=50$. (a) Retrieved estimations in XY plane. (b) Retrieved estimations in XZ plane.

choice of the test functions, which are derived from the family of analytic functions in the plane, the nonlinear source localization problem for systems characterized by wave equation can be obtained efficiently with a noniterative solution method deploying a proper annihilating filter.

The method achieves exact localization for the noiseless case. To deal with imperfect measurements due to noise and model mismatch, we proposed a denoising scheme inspired from Cadzow's composite property mapping algorithm. Preliminary results showed that the localization algorithm and the denoising scheme reach the theoretical limit paving the way for real applications.

In the current problem setting, the generating distribution is assumed to be a set of point acoustic sources which is a reasonable assumption for some sort of thermo/photo acoustic source imaging problems. However, it is a rather strong sparsity constraint to assume that the acoustic field on the measurement surface is generated by a superposition of $M$ point sources. Future research will focus on the modeling of the source distribution. Moreover, we also consider the possibility and feasibility of the proposed method for real applications such as ultrasound tomography and thermo/photo acoustic imaging.

\section{ACKNOWLEDGMENTS}

The authors would like to thank Dr. Djano Kandaswamy for his constructive remarks. This work was supported in part by the Swiss National Science Foundation (under the grants 205330-132808 and PP00P2-123438) and in part by the Center for Biomedical Imaging (CIBM) of the Geneva-Lausanne Universities and the EPFL.

\section{REFERENCES}

[1] Kandaswamy, D., Blu, T., and Van De Ville, D., "Analytic sensing: Noniterative retrieval of point sources from boundary measurements," SIAM Journal on Scientific Computing 31(4), 3179-3194 (2009).

[2] Ammari, H., Bossy, E., Jugnon, V., and Kang, H., "Mathematical modeling in photoacoustic imaging of small absorbers," SIAM Rev. 52, 677-695 (November 2010).

[3] Silva, C., Maltez, J. C., Trindade, E., Arriaga, A., and Ducla-Soares, E., "Evaluation of 11 and 12 minimum norm performances on eeg localizations," Clinical Neurophysiology 115(7), 1657 - 1668 (2004). 
[4] Lawson, C. L. and Hanson, R. J., [Solving Least Squares Problems (Classics in Applied Mathematics)], Society for Industrial Mathematics, new edition ed. (1987).

[5] De Peralta-Menendez, R. and Gonzalez-Andino, S., "A critical analysis of linear inverse solutions to the neuroelectromagnetic inverse problem," Biomedical Engineering, IEEE Transactions on 45, 440 -448 (april 1998).

[6] Ramrez, R. R., Wipf, D., and Baillet, S., "Neuroelectromagnetic source imaging of brain dynamics," in [Computational Neuroscience], Chaovalitwongse, W., Pardalos, P. M., and Xanthopoulos, P., eds., Springer Optimization and Its Applications 38, 127-155, Springer New York.

[7] Bouman, C. A., Webb, K. J., and Millane, R. P., "Nonlinear multigrid algorithms for bayesian optical diffusion tomography," IEEE Transactions on Image Processing 10(6), 909-922 (2001).

[8] Fannjiang, A., "The music algorithm for sparse objects: A compressed sensing analysis," CoRR abs/1006.1678 (2010).

[9] Eeg, F., Localization, M. S., Mosher, J. C., Mosher, J. C., Leahy, R. M., and Leahy, R. M., "Recursive music: A framework for eeg and meg source localization," (1998).

[10] Andrieux, S. and Abda, A. B., "Identification of planar cracks by complete overdetermined data: inversion formulae," Inverse Problems 12(5), 553 (1996).

[11] Zemp, R. J., "Photoacoustic imaging in biomedicine," in [CLEO:2011 - Laser Applications to Photonic Applications], CLEO:2011 - Laser Applications to Photonic Applications, JTuG1, Optical Society of America (2011).

[12] Natterer, F. and Wubbeling, F., [Mathematical methods in image reconstruction], Society for Industrial and Applied Mathematics, Philadelphia, PA, USA (2001).

[13] Blu, T., Dragotti, P.-L., Vetterli, M., Marziliano, P., and Coulot, L., "Sparse sampling of signal innovations," IEEE Signal Processing Magazine 25(2), 31-40 (2008).

[14] Vetterli, M., Marziliano, P., and Blu, T., "Sampling signals with finite rate of innovation," IEEE Transactions on Signal Processing 50(6), 1417-1428 (2002). IEEE Signal Processing Society's 2006.

[15] Isakov, V., "Uniqueness and stability in multi-dimensional inverse problems," Inverse Problems 9(6), 579 (1993).

[16] Sarason, D., [Complex function theory], American Mathematical Society, Providence, RI, second ed. (2007).

[17] Kandaswamy, D., Blu, T., and Van De Ville, D., "Analytic sensing: Direct recovery of point sources from planar Cauchy boundary measurements," in [Proceedings of the SPIE Conference on Mathematical Imaging: Wavelet XII], 6701, 67011Y-1-67011Y-6 (2007).

[18] Cadzow, J. A., "Signal enhancement: A composite property mapping algorithm," IEEE Trans. on Acoust., Speech, Signal Processing 36, 39-62 (1988).

[19] Pauluzzi, D. and Beaulieu, N., "A comparison of snr estimation techniques for the awgn channel," Communications, IEEE Transactions on 48, 1681 - 1691 (oct 2000).

[20] Kay, S. M., [Fundamentals of Statistical Signal Processing, Volume I: Estimation Theory (v. 1)], Prentice Hall, 1 ed. (Apr. 1993). 